\title{
CME Renal medicine (100621): self-assessment questionnaire
}

\author{
Edited by Ben Caplin and Tahseen Chowdhury
}

\section{SAQs and answers are ONLINE for RCP fellows and collegiate members}

The SAQs printed in the CME section can only be answered online to achieve external CPD credits. Any comments should be sent in via email only: clinicalmedicine@rcplondon.ac.uk

\section{Format}

Candidates are asked to choose the best answer from the five possible answers. This best of five format is used in many medical examinations, however the questions are not intended to be representative of those used in the MRCP(UK) Part 1 or Part 2 Written Examinations.

\section{The answering process}

1 Go to www.rcplondon.ac.uk/SAQ

2 Log on using your usual RCP username and password

3 Select the relevant CME question paper

4 Answer all 10 questions by selecting the best answer from the options provided

5 Once you have answered all the questions, click on Submit

\section{Registering your external CPD credits}

Carrying out this activity allows you to claim two external CPD credits. These will be automatically transferred to your CPD diary, where you can review the activity and claim your points.

1 According to UK Renal Registry data, what is the mortality rate of new starters on haemodialysis who are aged over 75 ?
(a) $30 \%$ in the first five years
(b) $30 \%$ in the first year
(c) $50 \%$ in the first five years
(d) $50 \%$ in the first year
(e) it varies too much to say.

2 The large increase in the number of older patients undergoing haemodialysis in the UK is largely due to which one of the following factors?

(a) broadening of dialysis criteria

(b) general practitioners (GPs) now record chronic kidney disease (CKD) stage in the patient record so diagnosis is made earlier (c) increased prevalence of glomerulonephritis in the elderly

(d) longer life expectancy on dialysis than in previous years

(e) shortage of transplant donor organs.

3 Which of the following anti-retroviral drugs is most frequently associated with development of a renal Fanconi syndrome in patients with HIV?
(a) abacavir
(b) dolutegravir
(c) lopinavir
(d) ritonavir
(e) tenofovir.

4 Which of the following statements regarding acute kidney injury (AKI) in patients with HIV is incorrect?

(a) Assessment of volume status and resuscitation is of paramount importance.

(b) Immune reconstitution inflammatory syndrome should be considered if a rapid rise in CD4 cell count occurs after initiation of anti-retroviral therapy.

(c) Renal biopsy is relatively contraindicated in HIV due to infection risk.

(d) Renal stone disease and obstruction should be considered in patients taking atazanavir.

(e) The classic triad of fever, rash and peripheral eosinophilia are often absent in AKI due to tubulointerstitial nephritis.

5 An asymptomatic 22-year-old female was referred with persistent dipstick positive haematuria following a routine medical examination. She previously had a normal urinary tract ultrasound. Her blood pressure was $110 / 75 \mathrm{mmHg}$, serum creatinine $84 \mu \mathrm{mol} / \mathrm{L}$ (normal range 70-100 $\mu \mathrm{mol} / \mathrm{L}$ ) and she had an undetectable urinary protein:creatinine ratio. She had no medical history of note and took no regular medications. On specific questioning she reported both parents are fit and well, however her maternal uncle needed a kidney transplant in his twenties and her elder sister has a young son who has recently been diagnosed with hearing difficulties and renal impairment.

\section{Which of the following statements are incorrect?}

(a) Genetic testing would be useful to determine the risk to her future offspring. 
(b) If she is a carrier for $\mathrm{X}$-linked Alport syndrome then her risk of kidney failure in later life is negligible, since she is a woman.

(c) She is likely to have a heterozygous COL4A5 mutation.

(d) She should be reviewed annually for the development of hypertension, renal impairment or proteinuria.

(e) Urinary dipstick screening should be offered to her family members.

6 Which of the following is not an inherited condition resulting from mutations in the genes encoding type-IV collagen, presenting with microscopic haematuria and associated with end-stage renal disease?

(a) autosomal recessive Alport syndrome

(b) hereditary angiopathy with nephropathy, aneurysms, and muscle cramps (HANAC) syndrome

(c) IgA nephropathy

(d) thin basement membrane nephropathy

(e) X-linked Alport syndrome.

7 Which one of the following is least likely to cause acute kidney injury (AKI)?
(a) aciclovir
(b) amphotericin B
(c) iodinated contrast
(d) piperacillin/tazobactam
(e) trimethoprim.

8 Which of the following are associated with haemolyticuraemic syndrome?
(a) anaemia
(b) diarrhoea
(c) elevated lactate dehydrogenase
(d) thrombocytopaenia
(e) all of the above.

9 Which one of the following statements is true regarding salt intake?

(a) Careful dietary history and diary keeping is the most accurate method of monitoring adherence to a low salt diet. (b) Government strategies to lower salt intake have no effect in lowering population salt intake.

(c) Lack of adherence to a low salt diet is primarily due to palatability.

(d) The average salt intake is more than five times the intake of our ancestors.

(e) The lowest salt intakes reduce blood pressure to the greatest extent.

10 Which one of the following statements is true in regards to salt intake in medical conditions?

(a) Cohort studies have found a linear relationship between estimated urinary sodium excretion and cardiovascular events.

(b) Salt restriction is safe in heart failure, particularly in those with high-dose diuretics.

(c) Salt restriction is the keystone of management in heart failure, based on large population-based studies.

(d) The benefit of salt restriction in chronic kidney disease is through its effect on blood pressure and urinary albumin excretion.

(e) There is concern regarding the enhancing effect of salt restriction and angiotensin receptor inhibitors.

\section{CME Emergencies in gastroenterology SAQ}

Answers to the CME SAQ published in Clinical Medicine October 2015

$\begin{array}{llllllllll}\text { Q1 } & \text { Q2 } & \text { Q3 } & \text { Q4 } & \text { Q5 } & \text { Q6 } & \text { Q7 } & \text { Q8 } & \text { Q9 } & \text { Q10 }\end{array}$
(b)
(c)
(c)
(d)
(e)
(a)
(e)
(a) (c) 